\title{
Design, Fabrication, and Testing of Micromachined Silicone Rubber Membrane Valves
}

\author{
Xing Yang, Charles Grosjean, and Yu-Chong Tai
}

\begin{abstract}
Technologies for fabricating silicone rubber membranes and integrating them with other processes on silicon wafers have been developed. Silicone rubber has been found to have exceptional mechanical properties including low modulus, high elongation, and good sealing. Thermopneumatically actuated, normally open, silicone rubber membrane valves with optimized components have been designed, fabricated, and tested. Suspended silicon nitride membrane heaters have been developed for low-power thermopneumatic actuation. Composite silicone rubber on Parylene valve membranes have been shown to have low permeability and modulus. Also, novel valve seats were designed to improve sealing in the presence of particles. The valves have been extensively characterized with respect to power consumption versus flow rate and transient response. Low power consumption, high flow rate, and high pressure have been demonstrated. For example, less than $40 \mathrm{~mW}$ is required to switch a 1-slpm nitrogen flow at 33 psi. Water requires close to $100 \mathrm{~mW}$ due to the cooling effect of the liquid. [427]
\end{abstract}

Index Terms - Parylene, silicone rubber, thermopneumatic actuation, valve.

\section{INTRODUCTION}

A number of active MEMS valves [1]-[5] have been demonstrated in the past. Although the configuration of these valves could be different (normally open or normally closed), the underlying structure is similar. In general, these devices consist of a deflectable membrane and a stationary valve seat with inlet/outlet. When the membrane is deflected and seals the inlet on the valve seat, the valve is closed. Given the similar structure, differences among the valves center on geometry, the actuation mechanism, and the membrane material. Low power, small size, and relatively large flow rates are desirable characteristics for a microvalve. To achieve high flow rate, a large gap is required between the seat and sealing membrane along with fairly large inlet/outlet holes. If a rigid membrane material is used, the size of the structure must be fairly large, or very high force must be used to deflect the membrane to the valve seat. If a rigid material is used for the valve seat and membrane, high force is required to ensure that the membrane conforms well to the sealing surface. Also, defects on either surface or particles can hinder effective sealing.

Manuscript received March 3, 1999; revised August 24, 1999. This work was supported by the DARPA MICROFLUMES program under Naval Ocean Systems Center Contract N66001-96-C-83632. Subject Editor, D. J. Harrison.

X. Yang is with Tanner Research, Inc., Pasadena, Ca 91107 USA.

C. Grosjean and Y.-C. Tai are with Caltech Micromachining Laboratory, Department of Electrical Engineering, California Institute of Technology, Pasadena, CA 91125 USA.

Publisher Item Identifier S 1057-7157(99)09617-1.
Another key respect of the microvalves is the actuation mechanism. Many actuation mechanisms, including thermal [1], electromagnetic [2], pneumatic [3], [4], and thermopneumatic [5], have been investigated for valve operations. It was found [5] that thermopneumatic actuation can provide large force through a long stroke. The actuation was achieved by heating/cooling a closed cavity, which was filled with a working liquid and sealed with a glass substrate heater on one side and a deflectable membrane on the other. Although good performance was demonstrated, the power consumption was fairly high due to the inefficiency of a simple resistive heater on a solid glass substrate in heating the working fluid. A large portion of the input power to the heater went into heating the glass heater substrate rather than heating the working fluid.

In this paper, we present our approach of using low modulus membrane materials to reduce the force required for closing the valve and improving sealing on the seat, and low power heater designs for thermopneumatic actuation to reduce heat lost to the environment.

\section{Silicone Rubber Membrane}

\section{A. Silicone Rubber MRTV 1}

It has been reported [3], [4] that spin-on silicone rubber can be used as a membrane material. Silicone rubber exhibits a very low modulus [3], good compatibility with IC processes, high elongation, and good sealing properties on rough surfaces. We have chosen silicone rubber MRTV 1 from American Safety Technologies, Inc., as the valve membrane material. MRTV 1 is an addition cure mold making room-temperaturevulcanizable (RTV) silicone. Among its important physical properties listed in Table I, two of them are very attractive for valve applications. First, it can sustain elongation as high as $1000 \%$, which means very large deflection can be achieved in valve membranes. Second, sealing of this material on rough surfaces can be very good due to its very low durometer (Shore A 24).

Various processing experiments have been performed on MRTV 1. It was found that MRTV 1 exhibits excellent adhesion to silicon, silicon dioxide, silicon nitride, and metals due to the presence of silane groups in the formulation. Cured films tend to tear before peeling. MRTV 1 is resistant to buffered hydrofluoric acid, positive photoresist developer, isopropyl alcohol, and oxygen plasma for short periods of time ( $<30 \mathrm{~min}$ ). Long-term exposure to strong acids, organic solvents such as acetone, xylene, benzene, and strong alkalis will destroy the films. The etch rate in $\mathrm{CF}_{4}$ or $\mathrm{SF}_{6}$ plasma is 
TABLE I

Physical Properties of Silicon Rubber MRTV 1

\begin{tabular}{l|l}
\hline Mixed Viscosity, cps & 60,000 \\
\hline Hardness, Durometer & Shore A 24 \\
\hline Tensile Strength, psi & 500 \\
\hline Tear Strength, Die B lb/in & 125 \\
\hline Tensile Elongation, \% & 1,000 \\
\hline Temperature Range, ${ }^{\circ} \mathrm{C}$ & -55 to 200 \\
\hline Thermal Conductivity, W/cm- ${ }^{\circ} \mathrm{C}$ & 0.002 \\
\hline Dielectric Strength, Volts/mil & 550 \\
\hline Volume Resistivity, ohm-cm & $1.6 \times 10^{15}$ \\
\hline
\end{tabular}

similar to silicon dioxide. Adhesion to photoresist and most glues is very poor. Thin silicone rubber films are permeable to chemical vapors and absorb some liquids.

\section{B. Membrane Fabrication}

A membrane fabrication process based on spin coating of MRTV 1 was developed and is shown in Fig. 1. First, a layer of 1 - $\mu$ m-thick low-stress low-pressure chemical vapor deposition (LPCVD) silicon nitride was deposited on the wafers. The wafer backside was patterned with $3 \times 3 \mathrm{~mm}^{2}$ windows using $\mathrm{SF}_{6} / \mathrm{O}_{2}$ plasma. $\mathrm{KOH}$ was then used to etch the silicon to the front side nitride forming $2.3 \times 2.3 \mathrm{~mm}^{2}$ membranes. MRTV 1 silicone was prepared by following the procedure suggested by the manufacturer and spun on the wafer front side, forming a $132-\mu \mathrm{m}$ silicone rubber layer. The silicone rubber was then cured at room temperature for $24 \mathrm{~h}$. Finally, the nitride membranes were removed using $\mathrm{SF}_{6} / \mathrm{O}_{2}$ plasma from backside leaving free silicone rubber membranes.

Although the spin-coating process is very convenient, it was found that MRTV 1 is sufficiently viscous at $60000 \mathrm{cps}$ that uniform spin coating can only be achieved at a very narrow range of spin rates. Experimentally, it was found that spin coating at the rate of $1000 \mathrm{rpm}$ for $80 \mathrm{~s}$ gives $132 \pm 1 \mu \mathrm{m}$-thick uniform layers of MRTV 1 on silicon wafers. Slower speeds result in uneven films while higher speeds streak, bubble, and redistribute fillers nonuniformly.

An alternative method to fabricate silicone rubber membrane is squeegee coating. The process is shown in Fig. 2. It started with $520-\mu \mathrm{m}$-thick wafers. A layer of $1.5-\mu \mathrm{m}$-thick silicon dioxide was grown and patterned on both sides. The wafers were then etched $235 \mu \mathrm{m}$ from both sides in $\mathrm{KOH}$, leaving a cavity on each side of the wafer and a $50-\mu \mathrm{m}$-thick silicon membrane. The top cavity was $4.8 \times 5.8 \mathrm{~mm}^{2}$ and served as a mold for the silicone rubber. The bottom cavity defined the dimensions of the silicone membrane, which was $1.5 \times 2.5 \mathrm{~mm}^{2}$. A layer of $0.5-\mu \mathrm{m}$-thick low-stress silicon nitride was deposited on both sides. Premixed MRTV 1 was then poured into the cavities on the wafer front side. By scraping a piece of glass with a flat smooth edge across the wafer, extra silicone rubber was taken away and a layer of

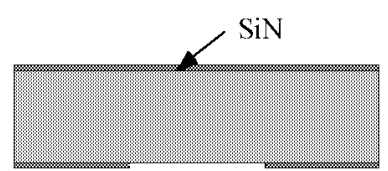

(a)

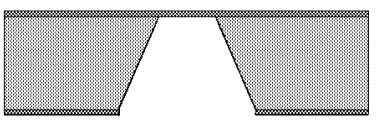

(b)

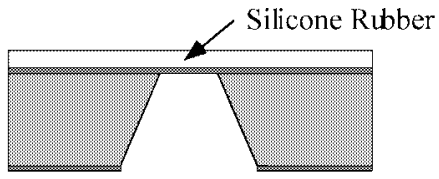

(c)

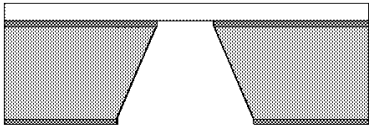

(d)

Fig. 1. Fabrication process for spin-coated silicone rubber membrane.
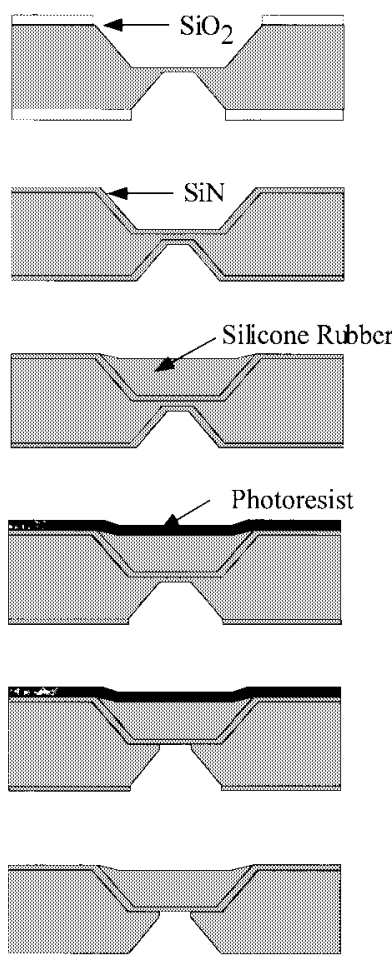

Fig. 2. Fabrication process for squeegee-coated silicone rubber membrane.

silicone rubber film was left in the cavity. The backside nitride was patterned using $\mathrm{SF}_{6} / \mathrm{O}_{2}$ plasma to remove the nitride over the silicon membrane. The $50-\mu \mathrm{m}$ silicon membrane was etched using $\mathrm{BrF}_{3}$ vapor [6], while the remainder of the wafer was protected with photoresist and silicon nitride. Last, the silicon nitride underneath the silicone rubber membrane was stripped from the backside.

The squeegee-coating technique produces uniform films from die to die. Compared to spin-coating, squeegee-coating 


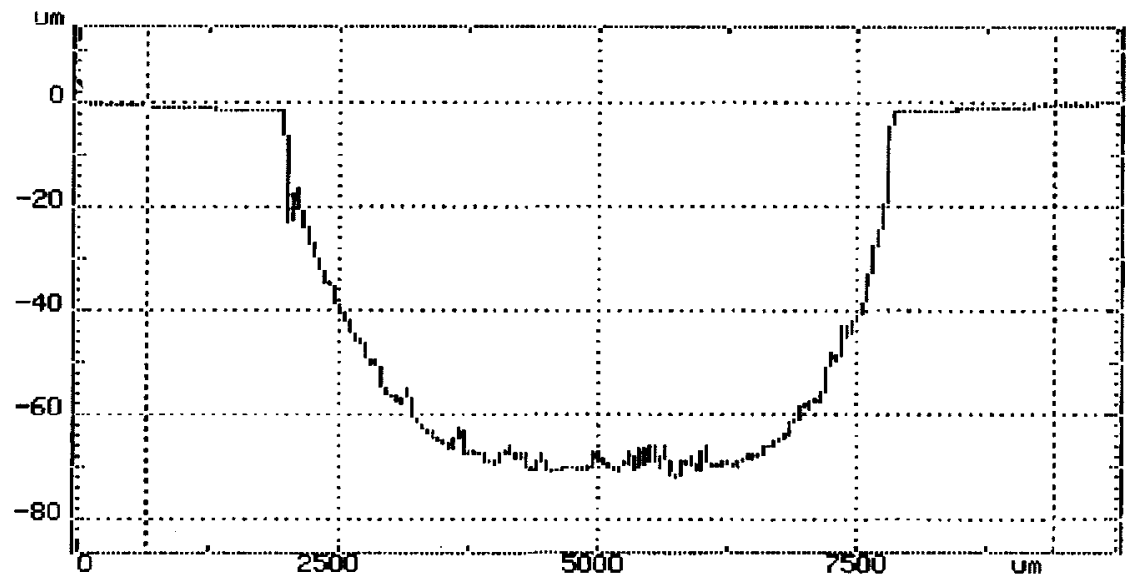

Fig. 3. Surface profile of a cured silicone rubber membrane.

can produce uniform films with various thicknesses by changing the depth of the cavity in the substrate. Also, only part of the wafer will be coated with silicone rubber while the rest of the wafer is exposed. This has the advantage that other fabrication processes such as bonding can still be achieved on the areas without silicone rubber.

It was found that the squeegee-coating process produces a recess at the center of the membrane due to surface tension at the corners of the cavity. Fig. 5 shows the profile of the silicone rubber layer along the $5.8-\mathrm{mm}$ dimension of the cavity. In this case, the recess was $70 \mu \mathrm{m}$. As shown later, this recess serves perfectly as the gap between the valve seat and membrane, which simplifies valve seat fabrication.

\section{Membrane Testing}

Load-deflection tests [7] were performed on the silicone rubber membranes to measure Young's modulus $E$ and residual stress $\sigma$. The silicone rubber membrane chip was mounted and sealed on a special holder and was then placed on the $x-y$ stage of an optical microscope with $z$-direction focus adjustment calibrated in $1-\mu \mathrm{m}$ increments. Pneumatic pressure was applied from the backside windows. By adjusting the input pressure and focus position, the center deflection of the membrane was measured with $\pm 1-\mu \mathrm{m}$ resolution. It was shown in [7] that for a rectangular membrane, the load-deflection relationship of the rectangular membrane can be expressed as

$$
P=\frac{C_{1} \sigma t h}{a^{2}}+\frac{C_{2} E t h^{3}}{a^{4}}
$$

where

$P \quad$ applied pressure;

$E \quad$ Young's modulus of the membrane material;

$\sigma$ residual stress in the membrane;

$v \quad$ Poisson's ratio of the membrane material;

$t \quad$ thickness of the membrane;

$h$ deflection of the membrane;

$n \quad a / b$.

For square membranes, $C_{1}$ and $C_{2}$ are calculated to be 3.04 and 2.55, respectively, assuming that Poisson's ratio of silicone rubber is 0.5 . Since Young's modulus $E$ and residual stress $\sigma$ independently appear in the linear term and the cubic term of

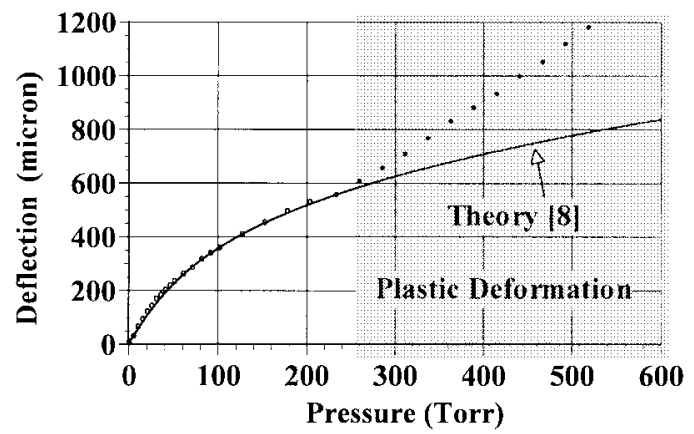

Fig. 4. Experimental results and theoretical fitting of silicone rubber membrane load-deflection testing.

(1), they can be determined by fitting the experimental data with (1).

Fig. 4 shows the measurement data and the fitting curve for a $2.3 \mathrm{~mm} \times 2.3 \mathrm{~mm} \times 132 \mu \mathrm{m}$ membrane. From the fitting results, it was found that silicone rubber has a Young's modulus of $0.51 \mathrm{MPa}$ and residual stress of $0.08 \mathrm{MPa}$.

It can be seen from Fig. 4 that very large deflection can be achieved in silicone rubber membrane with small pressure input. For example, at $14.6 \mathrm{psi}$, the membrane deflects $1.54 \mathrm{~mm}$, which corresponds approximately to a $115 \%$ increase in volume and a $100 \%$ increase in surface area. Fig. 5 shows the side view of the membrane at (a) 1, (b) 5, (c) 10, and (d) 15 psi.

Fig. 4 also shows that if pressure larger than 5.0 psi is applied to the membrane and the deflection of the membrane is more than $520 \mu \mathrm{m}$, the deflection data start to deviate from the theoretical model. Experimentally, it was found that the membrane was plastically deformed in this range. In other words, the membrane would not go back to the original flat position once the pressure was released. However, plastic deformation is not a concern to the valve because, as shown later, the design goal for the valve membrane stroke is less than $100 \mu \mathrm{m}$. More information on plastic deformation and reliability of the silicone rubber membrane can be found in [8].

Using these membranes, the sealing of silicone rubber on rough surfaces or surfaces with particles was also studied. In this experiment, a piece of glass was bonded on top of a squeegee-coated silicone rubber membrane. Before bonding, 


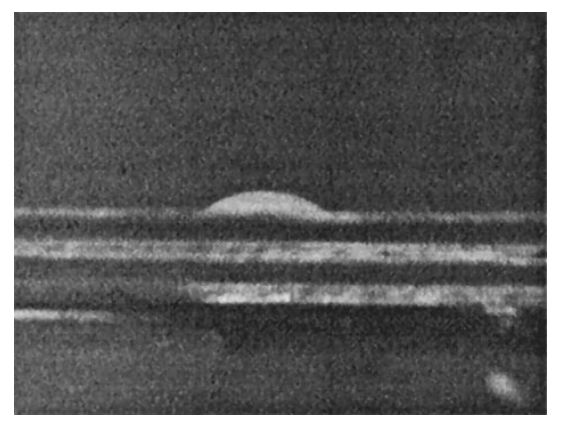

(a)

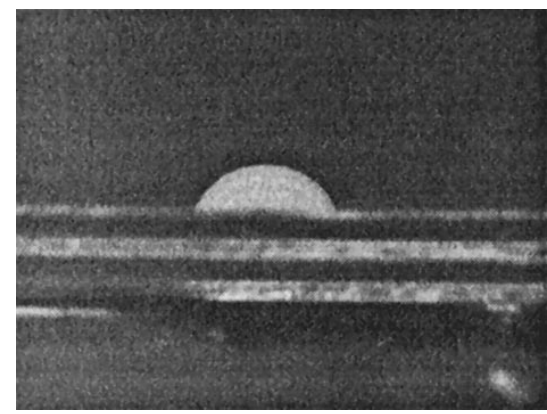

(b)

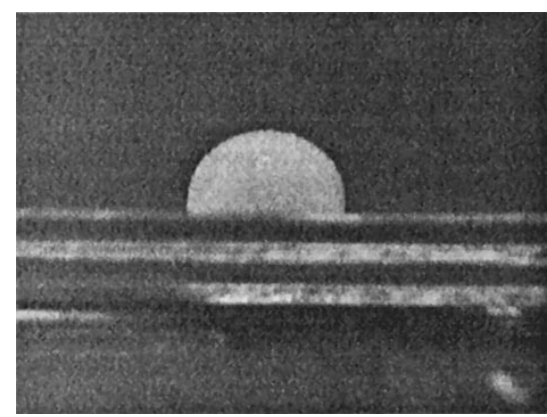

(c)

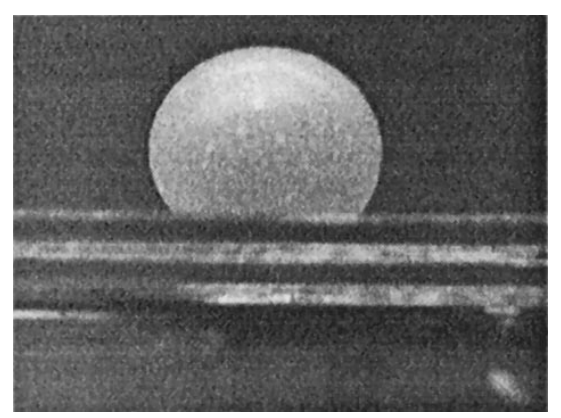

(d)

Fig. 5. Side view of silicone rubber membrane pneumatic actuation. Pressure load of (a) 1, (b) 5, (c) 10, and (d) 15 psi.

particles were purposely put on the side of the glass surface that the silicone rubber membrane would seal. Fig. 6 shows that with only 2-psi pneumatic pressure load applied to the membrane from the backside, the silicone rubber membrane deflected and fully encapsulated the particle on the glass surface. This demonstrates that, as an elastomer, silicone rubber possesses excellent sealing properties in the presence of particles. Good sealing is crucial to the valve because any nonsealed point on the valve seat would cause a significant leak rate when the valve is supposed to be closed.

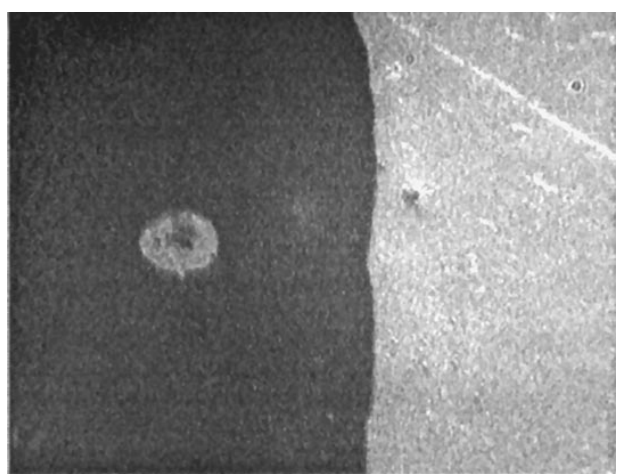

Fig. 6. Sealing testing of silicone rubber membrane. With 2-psi pressure input to the back, the silicone rubber membrane deflected and partially sealed against a piece of glass. On the left, the silicone rubber membrane fully encapsulated a particle that was put on the glass purposely.

It is known that silicone rubber is permeable to most fluids. Since the thermopneumatic actuation for valve operation is based on the expansion and/or evaporation of fluids in a sealed cavity, the permeability of silicone rubber could seriously affect the performance and lifetime of the valve. Experiments were performed to study the permeability of various liquids through silicone rubber membrane. A sealed cavity was fabricated by first filling the cavity with liquid and then bonding a piece of glass to the back of the silicone rubber membrane chip. Cavities filled with deionized (DI) water, isopropyl alcohol, and 3M Performance Fluids (industrial versions of Fluorinert) PF 5060 and PF 5070 were made and left at room temperature. It was found that most liquids escaped from the sealed cavity as vapor in a matter of days.

In summary, large deflection and good sealing properties have been demonstrated with the silicone rubber membrane. Experimentally, it was also found that the membrane is permeable to the liquids used for thermopneumatic actuation.

\section{Silicone Rubber/Parylene Composite Membrane}

In Section II, although good performance has been achieved with the silicone rubber membrane, the slow leakage of fluids through the silicone rubber membrane limits the application of thermopneumatic actuation in valves. The proposed solution to this problem is the use of a vapor barrier layer between the working fluid and the silicone rubber layer. Most IC compatible materials that are impermeable to the chosen working fluids have very high modulus, which defeats the purpose of a silicone rubber membrane valve. It was found that Parylene, which has a fairly low modulus, is an effective barrier.

\section{A. Parylene}

Parylene [9] is the generic name for members of a unique family of thermoplastic polymers. Three forms of this polymer are currently available, each with unique properties that suit it to particular applications. The basic member of the series, Parylene N, is poly-para-xylylene, a completely linear, highly crystalline material. Parylene C and D are produced from the same monomer modified by the substitution of a chlorine atom for one or two of the aromatic hydrogens. The common physical properties [9] of Parylene N, C, and D are listed in 
TABLE II

Physical Properties of PARYlene

\begin{tabular}{c|c|c|c}
\hline Properties & Parylene N & Parylene C & Parylene D \\
\hline Tensile Strength (psi) & $6,000-11,000$ & 10,000 & 11,000 \\
\hline Elongation to Break (\%) & $20-250$ & 200 & 10 \\
\hline $\begin{array}{c}\text { Water Absorption } \\
(\% \text { after } 24 \text { hours) }\end{array}$ & Less than 0.1 & Less than 0.1 & Less than 0.1 \\
\hline Melting Point $\left({ }^{\circ} \mathrm{C}\right)$ & 420 & 290 & 380 \\
\hline $\begin{array}{c}\text { Thermal Conductivity } \\
\text { at } 25^{\circ} \mathrm{C}\left(\mathrm{W} / \mathrm{m}^{\circ} \mathrm{C}\right)\end{array}$ & 0.126 & 0.084 & unavailable \\
\hline $\begin{array}{c}\text { Moisture Transmission at } 90 \% \\
\text { RH, } 37^{\circ} \mathrm{C}\left(\mathrm{g} \text {-mil } / 100 \text { in }{ }^{2} \cdot \mathrm{d}\right)\end{array}$ & 1.5 & 0.21 & 0.25 \\
\hline
\end{tabular}

Table II. Parylene has been explored as passivation material or dielectric material between multilevel metallization for the IC industry. The deposition materials, systems, and services are available from Specialty Coating Systems, Inc. (SCS) [9]. All the depositions in this work were performed on a Parylene deposition system (model PDS 2010 LABCOTER 1) from SCS. Among the three forms of Parylene, Parylene $\mathrm{C}$ was chosen as the vapor barrier layer because of its low permeability to moisture and gases as seen from Table II.

\section{B. Mechanical Properties of Parylene C}

To measure the mechanical properties of Parylene C, 6.87$\mu \mathrm{m}$-thick Parylene $\mathrm{C}$ membranes in the dimensions of a $3.66 \times$ $3.76 \mathrm{~mm}^{2}$ have been fabricated. Similar load-deflection tests as described in Section II-C were performed on these Parylene C membranes. By fitting the experimental data, Young's modulus and residual stress of the Parylene $\mathrm{C}$ layer were found to be $4.48 \mathrm{GPa}$ and $20.88 \mathrm{MPa}$. This confirms that Parylene C has a low Young's modulus and suggests that it is possible to fabricate a fairly soft silicone rubber/Parylene composite membrane.

\section{Composite Membrane Fabrication}

Composite silicone rubber/Parylene membranes were fabricated using a process similar to the squeegee-coating process in Section II-B. To keep the membranes soft, a very thin layer of Parylene was used compared to the silicone thickness. As it was suspected that silicone absorbed the working fluid, the Parylene layer was sandwiched between the silicone rubber layer and the fluid. The process is shown in Fig. 7. It started with thermal oxidation of the wafers followed by patterning etching windows on both sides of the wafers. The wafers were then time etched in a $\mathrm{KOH}$ solution to form two cavities. The $520-\mu \mathrm{m}$-thick wafers were etched $235 \mu \mathrm{m}$ on each side in $\mathrm{KOH}$ leaving a $50-\mu \mathrm{m}$-thick silicon membrane. A layer of 0.5 - $\mu \mathrm{m}$-thick low-stress silicon nitride film was deposited on both sides, and the silicon nitride on the backside was removed using $\mathrm{SF}_{6} / \mathrm{O}_{2}$ plasma. The wafers were put back into $\mathrm{KOH}$ to etch away the silicon layer and a freestanding silicon nitride membrane was formed. Then, the wafer surface was roughened

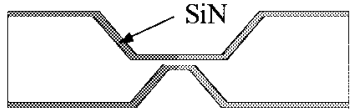

(a)

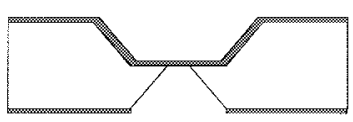

(b)

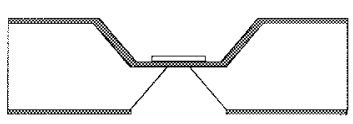

(c)

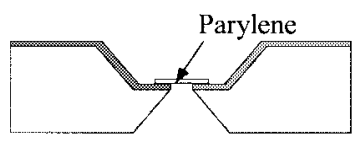

(d)

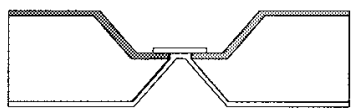

(e)

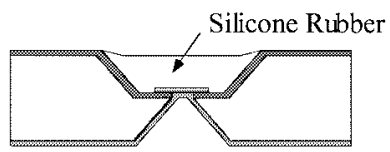

(f)

Fig. 7. Composite silicone rubber/Parylene fabrication process.

using $\mathrm{SF}_{6} / \mathrm{Ar}$ plasma followed by priming of A-174 Parylene adhesion promoter. A layer of $2-\mu \mathrm{m}$-thick Parylene $\mathrm{C}$ was deposited and patterned at the bottom of the top cavity. Then, a layer of MRTV 1 silicone rubber was molded into the top cavity by squeegee coating. On the backside, silicon nitride was patterned using $\mathrm{SF}_{6} / \mathrm{O}_{2}$ plasma to free the membrane. Finally, another layer of $2-\mu \mathrm{m}$-thick Parylene $\mathrm{C}$ was deposited on the backside to strengthen the edge of the membrane while the front side of the membrane was protected with dummy wafers in the deposition chamber. Due to the poor adhesion between silicone rubber and Parylene, the Parylene layer at the bottom of the top cavity had to be patterned to open the region for the silicone rubber to anchor to the silicon nitride surface.

A load deflection test was performed on the composite membrane. The results shown in Fig. 8 confirms that the composite membrane is still fairly flexible. For example, the membrane deflects $134 \mu \mathrm{m}$ at a pressure input of $4.1 \mathrm{psi}$. It also should be noticed that the composite membrane would be plastically deformed if the deflection is more than $200 \mu \mathrm{m}$ and pressure is higher than 6 psi.

Similar permeability testing as described in Section II-C has been performed on the composite membrane. Fluids were sealed in cavities formed by bonding a piece of glass to the backside of the composite silicone rubber/Parylene membrane. It was found from experiments that up to one month, no volume change and bubble formation were observed in the sealed cavities. This confirms that Parylene is an effective vapor barrier layer. 


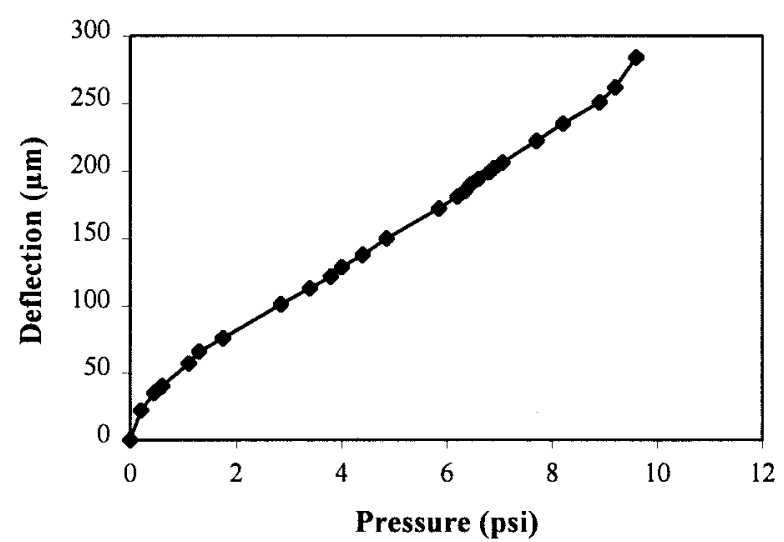

Fig. 8. Load-deflection testing of a composite silicone rubber/Parylene membrane.

It can be concluded from the above experiments that the silicone rubber/Parylene composite membrane is suitable for valve applications. Both Parylene and silicone rubber have been shown to have low modulus. By keeping Parylene layer thin, a flexible composite membrane can be made. While Parylene is used as the vapor barrier layer, the silicone rubber layer serves two purposes. First, it provides good sealing properties. Second, because of its low thermal conductivity, the silicone rubber layer minimizes the heat loss to the valve surrounding structures and hence reduces the power consumption of the valve operation. This will be shown in Section IV.

\section{THERMOPNEUMATIC ACTUATION}

\section{A. Freestanding Silicon Nitride Membrane Heater}

In the past, simple resistive heater on a glass substrate was used for thermopneumatic actuation. It was shown that such a heater consumes a lot of power because of its poor thermal efficiency. Computer simulation [10] shows that $98 \%$ of the heat generated by such a heater is lost through the glass substrate rather than heating the working fluid. To improve the thermal insulation, a gold heater on freestanding silicon nitride membrane has been developed. Because the membrane is thin and silicon nitride has a very low thermal conductivity, the heat loss would be significantly reduced. Gold is chosen as the heater material because it has high resistance to oxidation and low thermal mismatch with silicon nitride. Large thermal mismatch could potentially fracture the heater when the temperature at the surface of the heater gets high.

The fabrication process for the freestanding silicon nitride membrane heater starts with $0.5-\mu$ m-thick LPCVD low-stress silicon nitride deposition on the wafers. Then, the silicon nitride layer on the backside was patterned to open up the etching windows. The wafers were etched in a $\mathrm{KOH}$ solution from the backside until only a $20-\mu$ m-thick silicon layer was left. After that, a layer of $100-\AA \mathrm{Cr} / 5000-\AA \mathrm{Au}$ was evaporated and patterned to define the heater. An array of $200-\mu \mathrm{m}-$ diameter holes was etched in the silicon nitride membrane using $\mathrm{SF}_{6} / \mathrm{O}_{2}$ plasma. Last, the wafers were diced and put back into $\mathrm{KOH}$ solution to remove the $20-\mu \mathrm{m}$ silicon layer to free the membranes. Dicing before the structure releasing avoided the potential damage of the membranes during dicing.

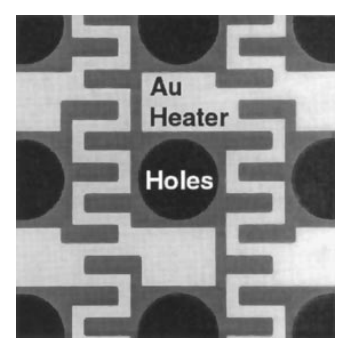

Fig. 9. Picture of a fabricated freestanding silicon nitride membrane heater.

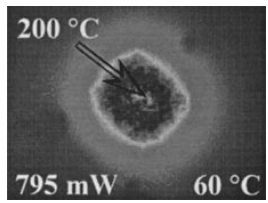

(a)

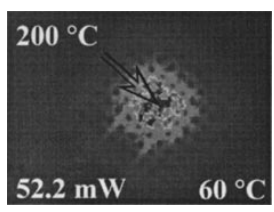

(b)

Fig. 10. Temperature profiles of heaters measured using Infrascope. (a) Glass substrate heaters. (b) Silicon nitride membrane heater.

Fig. 9 shows a fabricated heater. The array of holes equalizes the pressure across the membrane during the valve operation. Also, the gold square blocks between holes increase the heat conduction area and improve the frequency response of the thermopneumatic actuation.

To compare the performance of the silicon nitride membrane heater and the glass substrate heater, the temperature distribution on the heater surface was measured using an infrared microscope (Infrascope, Quantum Focus Instruments Corp.). Infrascope is a microthermal infrared imaging system with 3$\mu$ m spatial resolution and $0.1{ }^{\circ} \mathrm{C}$ temperature resolution. Under the Infrascope, heaters were mounted on a metal substrate using conductive adhesive. Fig. 10 shows measured temperature profile on the surface for the two types of heater. The silicon nitride membrane heater surface reached $200{ }^{\circ} \mathrm{C}$ with only 52.2-mW power input while the glass heater required 795$\mathrm{mW}$ power input for the same temperature. It should also be noticed that on the freestanding silicon nitride membrane, the heat is far more localized resulting in less loss via conduction to the surroundings. This confirms that compared to the glass substrate heater, the freestanding silicon nitride membrane heater has better thermal isolation and consumes less power.

During valve operation, the heater is sealed in a fluidfilled cavity. Because of the different thermal environment, the temperature on the heater surface would be different from the temperature on the same heater measured using Infrascope, even if the same power is applied to the heater. The average temperature across the heater surface during valve operation can be estimated if the temperature coefficient of resistance (TCR) of gold heaters is known. Experiments were performed to calibrate the TCR of gold. Two gold heaters on silicon nitride membrane with different designs 


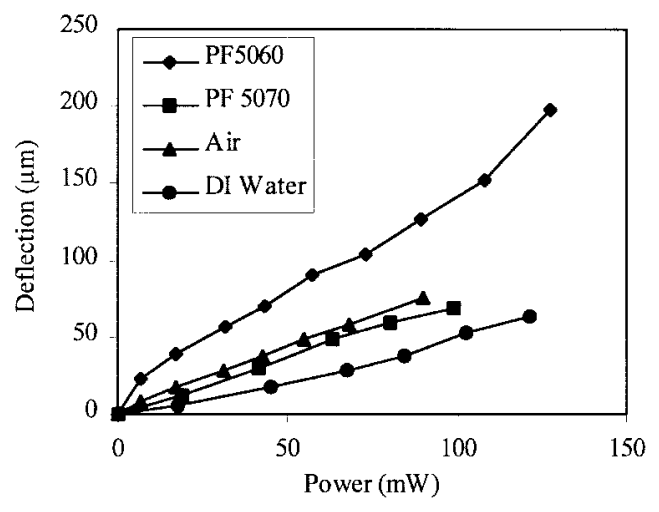

Fig. 11. Steady-state thermopneumatic actuation with various working fluids.

were set up in a temperature-controlled environment chamber. By slowly increasing the temperature and measuring the corresponding resistance change, the resistance change as a function of temperature was obtained. Enough time was given between two data points to allow the heaters to reach thermal equilibrium. By fitting the data with a linear function, the slope gave the TCR of gold, which was $0.003 /{ }^{\circ} \mathrm{C}$.

\section{B. Steady-State Response of Thermopneumatic Actuation}

To study the steady-state response of thermopneumatic actuation with various working fluids, a variety of actuators were assembled by bonding the silicon nitride membrane heater to the bottom of the composite membrane chip and sealing the formed cavity with a silicon or glass backing plate. The bonding and sealing were achieved with adhesive. Actuation was studied by applying power to the heater and measuring the corresponding deflection of the membrane from its rest position under a microscope with a calibrated focus. Enough time was given between two data points to allow the actuator to reach thermal equilibrium.

Using the composite membrane chip with a $3 \times 3 \mathrm{~mm}^{2}$ backside opening, heaters with three different dimensions $\left(2.9 \times 2.9 \mathrm{~mm}^{2}, 2.1 \times 2.1 \mathrm{~mm}^{2}\right.$, and $\left.1.1 \times 1.1 \mathrm{~mm}^{2}\right)$ were fabricated to investigate the effect of heater surface area on performance. The small heater $\left(1.1 \times 1.1 \mathrm{~mm}^{2}\right)$ and medium heater $\left(2.1 \times 2.1 \mathrm{~mm}^{2}\right)$ had similar steadystate performance with the small heater taking longer to reach thermal equilibrium. The large $\left(2.9 \times 2.9 \mathrm{~mm}^{2}\right)$ heater required significantly more power for a similar deflection, leading to the conclusion that more power was being lost to the substrate due to the proximity of the heater to the side walls.

Various fluids were sealed in the actuator and their thermopneumatic actuation performance was studied. Fig. 11 shows the results of power versus deflection for cavities fully filled with water, PF 5060, PF 5070, isopropanol, and air. Actuators with an air bubble in the cavity had higher power consumption and failed quickly due to overheating. With the same power input to the heater, the actuator sealed with PF 5060 achieved the highest deflection among all the fluids.

By using the TCR of gold, the average temperature across the heater surface was estimated and plotted as a function of power input for different working fluids. The results are shown

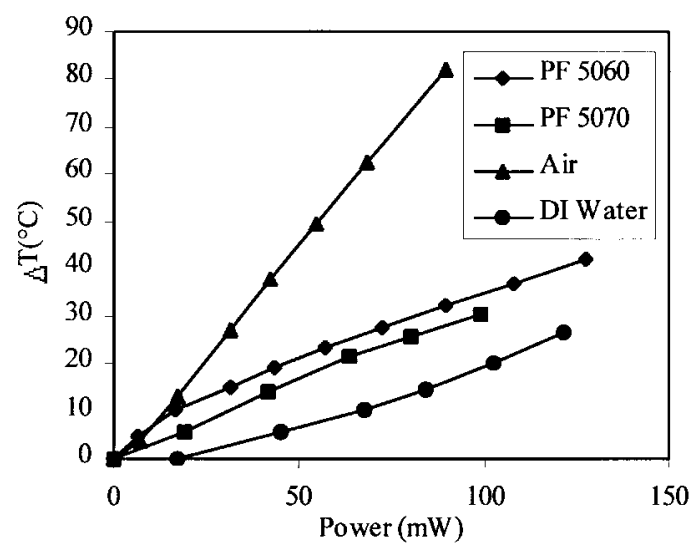

Fig. 12. Temperature increase on the heater surface during thermopneumatic actuation.

TABLE III

Time Constants of Thermopneumatic ACTUATION UNDER VARIOUS CONDITIONS

\begin{tabular}{c|c|c|c|c}
\hline Condition & Power $(\mathrm{mW})$ & Deflection $(\mu \mathrm{m})$ & $\begin{array}{c}\text { Heating Time } \\
\text { Constants (s) }\end{array}$ & $\begin{array}{c}\text { Cooling Time } \\
\text { Constants (s) }\end{array}$ \\
\hline PF 5060 & 42.5 & 45 & $1.7,38.2$ & $2.1,37.0$ \\
\hline $\begin{array}{c}\text { PF5060 (On } \\
\text { Heat Sink) }\end{array}$ & 42.5 & 33 & $1.5,7.1$ & $1.8,9.4$ \\
\hline PF 5070 & 40 & 30 & $2.5,32.0$ & $2.7,32.0$ \\
\hline DI Water & 93 & 43 & $1.6,45.8$ & $1.7,45.8$ \\
\hline Air & 50 & 35 & $1.3,40.7$ & $1.2,46.2$ \\
\hline
\end{tabular}

in Fig. 12. As expected, the low thermal conductivity of air results in very high temperatures.

\section{Transient Response of Thermopneumatic Actuation}

Transient performance was studied by driving the assembled thermopneumatic actuator with a square wave from a function generator while measuring membrane deflection as a function of time with a laser interferometer (Model PD-1000, PrecisionDynamics, Inc.). The response typically exhibits two time constants, a short one associated with heating the working liquid and a longer one related with the heating of the package and surroundings. A heater with good thermal insulation consumes less power, but it takes longer for the heat to dissipate, resulting in a tradeoff between power consumption and speed. While a valve can be closed quickly by applying a large power pulse, the opening time may be increased substantially due to the time to dissipate extra energy to the environment. Table III shows the measured time constants of actuators filled with various fluids. Air provides the fastest time constants due to its low density, even though its thermal conductivity is fairly low compared to the liquids. Using PF 5060 as the working fluid, the time constants are 1.7 and $38.2 \mathrm{~s}$ for heating and 2.1 and $37.0 \mathrm{~s}$ for cooling. As shown in Fig. 13, the second time constant can be improved substantially by using a metal heat sink on the package to 1.5 and $7.1 \mathrm{~s}$ for heating and 1.8 and $9.4 \mathrm{~s}$ for cooling at the expense of increased power consumption. 


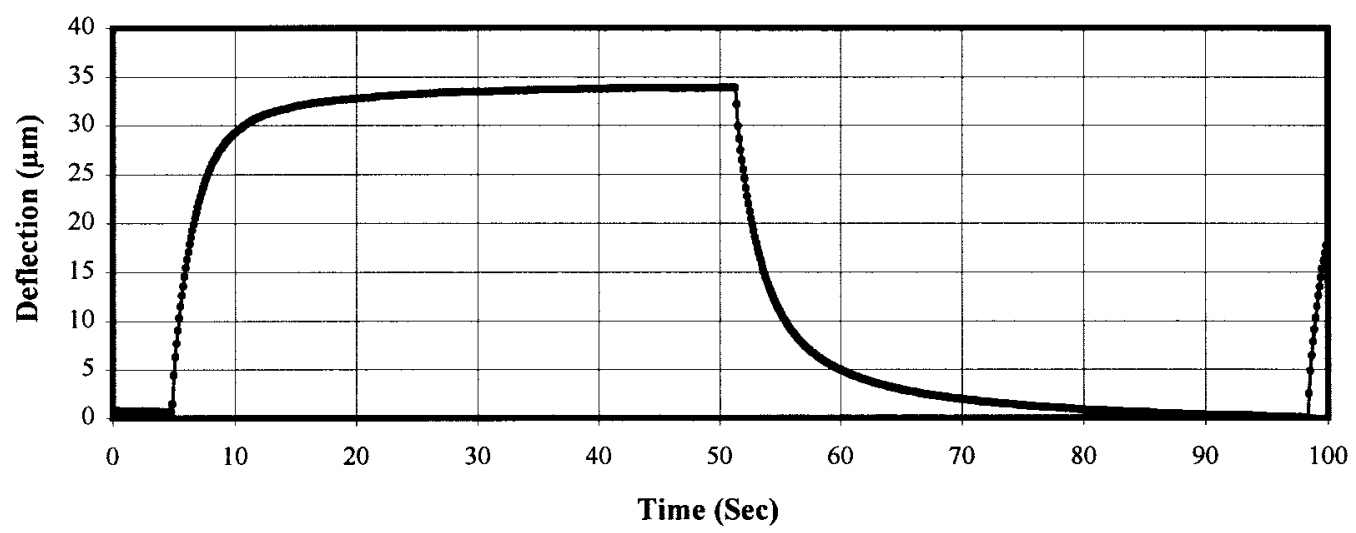

Fig. 13. Thermopneumatic actuation transient response.

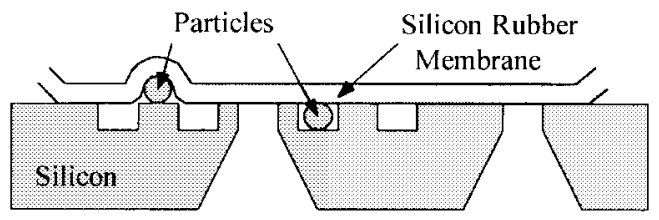

Inlet/Outlet

(a)

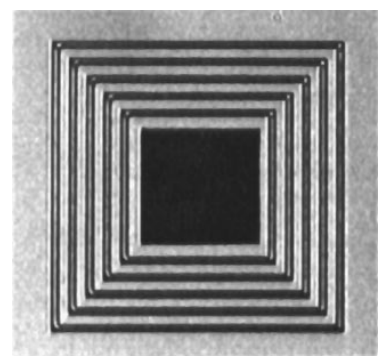

(b)

Fig. 14. Novel corrugated valve seat. (a) Valve seat design. (b) Picture of a fabricated valve seat.

\section{Thermopneumatic VALVE}

\section{A. Corrugated Valve Seat}

We have developed a new corrugated valve seat that complements the sealing properties of the silicone rubber membrane to reduce the leak rate of the valve. As shown in Fig. 14, the new valve seat has an array of concentric grooves 30 $\mu \mathrm{m}$ wide and $20 \mu \mathrm{m}$ deep around the inlet/outlet holes. The grooves was etched into the silicon substrate by $\mathrm{SF}_{6} / \mathrm{O}_{2}$ plasma. These grooves reduce the chance of the particles' sticking near the inlet/outlet. Also, by taking advantage of the elastomeric properties of silicone rubber, these grooves serve as redundant sealing rings so that even when there are particles, the silicone rubber could fully encapsulate the particles without compromising the performance of the valve. Valve seats with 400 - and $600-\mu \mathrm{m}$ square inlet/outlet were fabricated. Fig. 14(b) shows a fabricated valve seat.

\section{B. Flow Performance}

As shown in Fig. 15(a), valves were made by assembling a valve seat die, membrane chip die, heater die, and backing plate. Flexible plastic Tygon tubes (i.d. 1/32 in, o.d. 3/32 in)

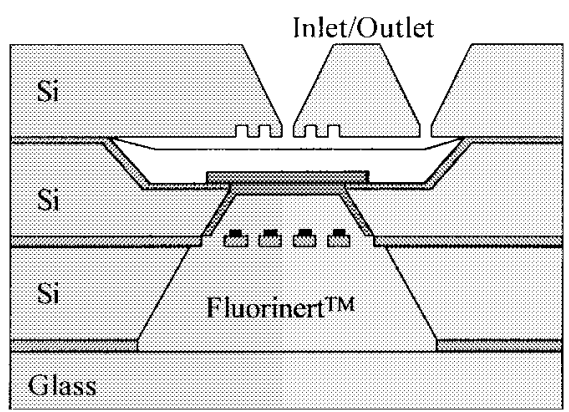

(a)

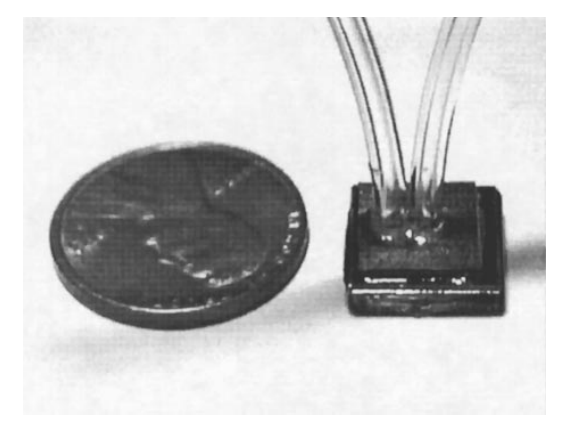

(b)

Fig. 15. Thermopnematic valve with composite silicone rubber/Parylene membrane. (a) Cross-sectional view. (b) Picture of an assembled valve.

were attached to valve inlet/outlet with epoxy. Fig. 15(b) is a picture of a fully packaged valve. As suggested by the thermopneumatic actuation results from Section III, all the valves were fully filled with PF 5060.

The flow performance of the valve was studied by measuring the flow rates at different pressure drops across the valve without power input to the heater. To investigate the effect of inlet/outlet hole size on the valve performance, valves with 400 $\times 400 \mu \mathrm{m}^{2}$ and $600 \times 600 \mu \mathrm{m}^{2}$ inlet/outlet holes were made and tested with nitrogen and water flow. The results varied due to the misalignment of the manual assembling process. Typical results are plotted in Fig. 16. The water flow rate through the valve is roughly 100 times smaller than the flow rate of nitrogen because water has a 100 times higher viscosity.

\section{Valve Performance with Nitrogen Flow}

Valve performance was tested with nitrogen flow. Compressed nitrogen and a pressure regulator were used to provide 


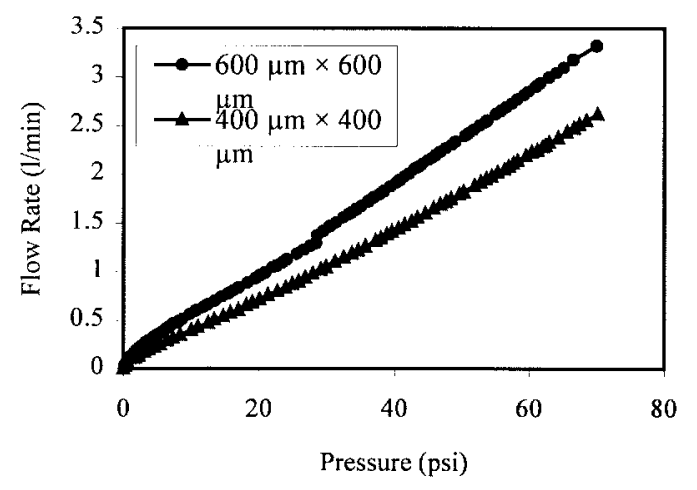

(a)

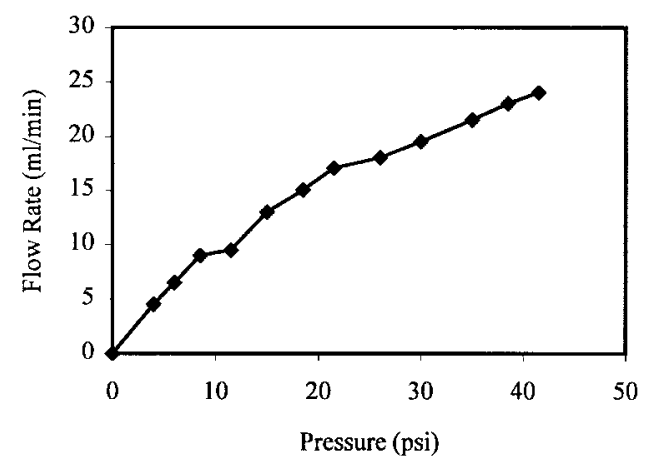

(b)

Fig. 16. Valve flow resistance with (a) nitrogen and (b) water.

flow up to $5 \mathrm{~L} / \mathrm{min}$ and pressure up to 75 psi. An HP $6115 \mathrm{~A}$ precision power supply was connected to the heater of the valve, and the current was measured with an HP 34401A multimeter. Pressure and flow rate were measured with a pressure sensor (Omega, Model HHP 4100, 0.1-psi resolution) and a flow meter (Cole Parmer, Model 32916, 0.01-L/min resolution).

The valve performance can be affected by several factors, including the alignment of the seat to the membrane, variations of inlet/outlet hole size, and choice of inlet ports (center or side of the valve seat chip). Misalignment will increase the power necessary to seal the valve for a given flow, as the contact area between the membrane and seat must be larger. As the valve seat is silicon, it is difficult to optically verify alignment after assembly. Flow testing of small and large hole size (400 and $600 \mu \mathrm{m}$ square, respectively) and valve seats confirmed the greater flow capability of the larger hole sizes, but it was determined that the increased flow was not necessary, especially at the price of increased power consumption. It was also found that valves were most efficient when the outlet was located at the center of the valve seat.

Typically, for a valve with $400 \times 400 \mu \mathrm{m}^{2}$ inlet/outlet and fully filled with PF 5060, the power required to close the valve usually varied between 40 and $70 \mathrm{~mW}$ at an inlet pressure of about $30 \mathrm{psi}$ and a flow rate of about $1 \mathrm{~L} / \mathrm{min}$. The lowest power consumption achieved is shown in Fig. 17(a). In this case, $35.5 \mathrm{~mW}$ was sufficient to shut off a flow of $1.04 \mathrm{~L} / \mathrm{min}$ at the inlet pressure of $33 \mathrm{psi}$. By using the measured TCR of gold, the average temperature on the heater surface was estimated to be $20^{\circ} \mathrm{C}$ above room temperature. Another valve

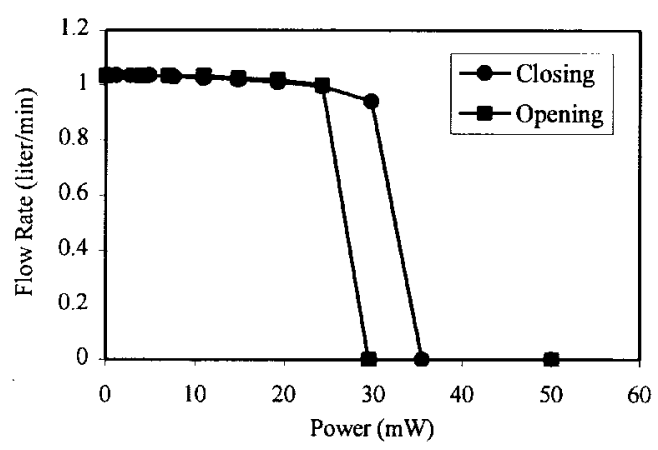

(a)

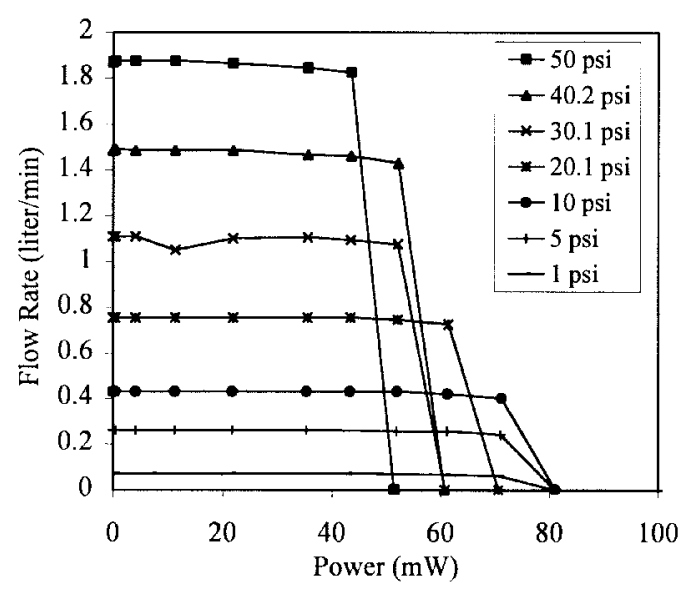

(b)

Fig. 17. Thermopneumatic valve performance with nitrogen flow.

that had similar construction, except for a 1-mm-size bubble in the actuation cavity, required a power of $139 \mathrm{~mW}$ to close valve under the similar testing conditions. At that power input, the average temperature at the heater surface was found to be $50{ }^{\circ} \mathrm{C}$ above room temperature.

Another data set was taken for a single valve with a wide range of inlet pressure and is shown in Fig. 17(b). This valve exhibits higher power consumption, which is most likely due to valve seat/membrane misalignment. Of interest is the trend of increasing power consumption for lower inlet pressure. The most likely explanation of this behavior is increased contact area between the membrane and valve seat, resulting in more heat loss by conduction through the membrane. This, along with the hysteresis between opening and closing powers, indicates that improved thermal insulation should be considered for the membrane, possibly by making the silicone rubber layer thicker, which would not significantly affect the stiffness due to the Parylene layer.

\section{Valve Performance with Water Flow}

Valves were also tested with water. The test setup was similar to the nitrogen testing setup except that a water reservoir pressurized with compressed nitrogen served as water source. The water pressure was regulated by monitoring the pressure of compressed nitrogen. Inlet pressure to the valve was measured with a water pressure sensor, while the flow rate was measured with a graduated cylinder and a stopwatch. 


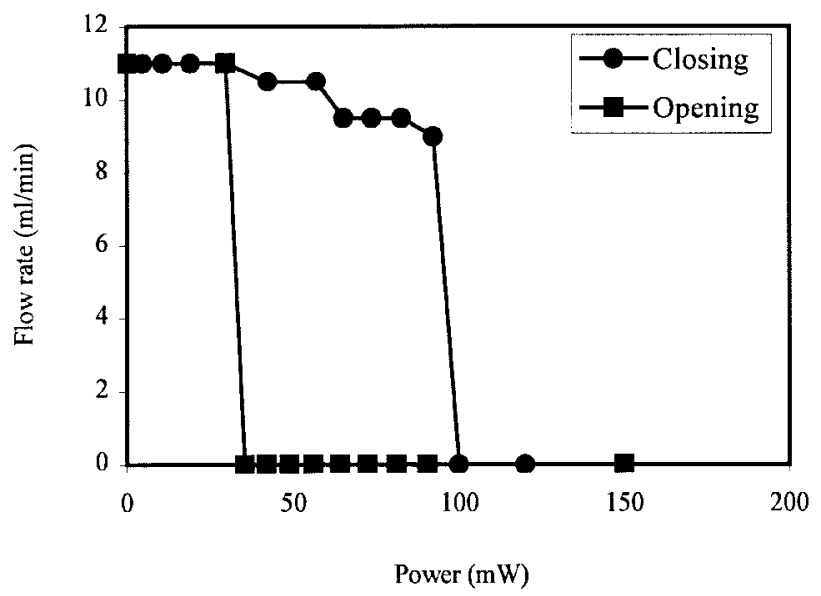

Fig. 18. Thermopneumatic valve performance with water flow at inlet pressure of 32 psi.

Fig. 18 shows testing results with inlet pressure of 32 psi and flow rate of $11 \mathrm{ml} / \mathrm{min}$. The valve used in this case was the same one that achieved the lowest power consumption for nitrogen flow. In the case of water flow, it took $100 \mathrm{~mW}$ of power to close the valve, and at that power input, the average temperature on the heater surface was $35{ }^{\circ} \mathrm{C}$ above room temperature. For the same inlet pressure, it required an extra $65 \mathrm{~mW}$ of power to close the water flow versus the nitrogen flow. Computer simulations show that the hysteresis between opening and closing power is mainly caused by convection heat loss to the liquid. This also explains that when the valve is tested with water and nitrogen, the opening power is similar but the closing power of water flow is much higher.

\section{CONCLUSION}

Normally open low-power thermopneumatic MEMS valves utilizing low modulus membranes have been designed, fabricated, and tested. A novel suspended heater has been developed to reduce heat loss. By using a "soft" membrane with a large gap, and thermopneumatic actuation, high flow rates are achievable while still keeping power consumption low. A new valve seat has been developed that complements sealing properties of the silicone membrane to reduce the leak rate of the valve when closed. The steady state and transient behavior of the thermopneumatic actuation have been studied and an optimum working fluid and heater design have been identified. The valve shows very low power consumption. A 1-slpm nitrogen flow with an inlet pressure of 33 psi can be controlled with less than $40 \mathrm{~mW}$. A water flow at similar pressure requires $100 \mathrm{~mW}$ due to additional heat lost by convection. The valve structure measures $8 \mathrm{~mm} \times 8 \mathrm{~mm} \times 2 \mathrm{~mm}$ and satisfies the requirements of small size, low power, and high flow rate.

\section{ACKNOWLEDGMENT}

The authors would like to thank E. Meng for help with testing, X.-Q. Wang for Parylene processing, Dr. Q. Lin for simulation, and $\mathrm{T}$. Roper for assistance with fabrication.

\section{REFERENCES}

[1] P. W. Barth, "Silicon microvalve for gas flow control," in Int. Conf. Solid-State Sensors and Actuators Dig. Tech. Papers, Stockholm, Sweden, June 1995, vol. 2, pp. 276-279.

[2] C. Ahn, T. Henderson, W. Heineman, and B. Halsall, "Development of a generic microfluidic system for electrochemical immunoassaybased remote bio/chemical sensors," in Proc. $\mu$ TAS'98 Workshop, Banff, Canada, Oct. 1998, pp. 225-230.

[3] L. Bousse, E. Dijkstra, and O. Guenat, "High density array of valves and interconnects for liquid switching," in Solid State Sensor and Actuator Workshop Tech. Dig., Hilton Head Island, SC, June 1996, pp. 272-275.

[4] C. Vieider, O. Ohman, and H. Elderstig, "A pneumatically actuated microvalve with a silicone rubber membrane for integration with fluid handling systems," in Int. Conf. Solid-State Sensors and Actuators Dig. Tech. Papers, Stockholm, Sweden, June 1995, vol. 2, pp. 284-286.

[5] M. Zdeblick, R. Anderson, J. Janskowski, B. Kline-Schoder, L. Christel, R. Miles, and W. Weber, "Thermopneumatically actuated microvalves and integrated electro-fluidic circuits," in Solid State Sensors and Actuator Workshop Tech. Dig., Hilton Head Island, SC, June 1994, pp. 251-255.

[6] X. Q. Wang, X. Yang, K. Walsh, and Y. C. Tai, "Gas phase silicon etching with bromine trifluoride," in Int. Conf. Solid-State Sensors and Actuators Dig. Tech. Papers, Chicago, IL, June 1997, vol. 2, pp. $1505-1508$.

[7] O. Tabata, K. Kawahata, S. Sugiyama, and I. Igarashi, "Mechanical property measurements of thin films using load deflection of composite rectangular membranes," Sens. Actuators, vol. A20, pp. 135-141, 1989.

[8] C. Grosjean, G. B. Lee, W. Hong, Y. C. Tai, and C. M. Ho, "Micro balloon actuators for aerodynamic control," in Proc. Int. Workshop Micro Electro Mechanical Systems, Heidelberg, Germany, Jan. 1998, pp. $166-171$

[9] "Parylene conformal coatings specifications and properties," Specialty Coating Systems, Inc., Indianapolis, IN 46241.

[10] P. L. Bergstrom, J. Ji, Y.-N. Liu, M. Kaviany, and K. D. Wise, "Thermally driven phase-change microactuation," J. Microelectromech. Syst., vol. 4, no. 1, pp. 10-17, 1995.

Xing Yang received the B.S. degree in applied physics from Beijing University of Posts and Telecommunications, China, in 1991 and the M.S. and Ph.D. degrees in electrical engineering from the California Institute of Technology, Pasadena, in 1994 and 1998, respectively.

He was a Research Scientist working on MEMS at ACLARA BioSciences $\mathrm{He}$ is currently a Scientist working on MEMS at Tanner Research, Inc., Padadena, CA. His current research interests are microfluidic devices and systems and polymer MEMS

Charles Grosjean received the B.S. degree in engineering and applied science in 1994 and the M.S. degree in electrical engineering from the California Institute of Technology, Pasadena, in 1995, where he currently is pursuing the Ph.D. degree in the Caltech Micromachining Laboratory.

His research interests include alternative materials and their application in optical and microfluidic devices.

Yu-Chong Tai received the B.S. degree in electrical engineering from the National Taiwan University, R.O.C., in 1981 and the M.S. and Ph.D. degrees in electrical engineering from the University of California at Berkeley in 1986 and 1989 , respectively.

He joined the Faculty of Electrical Engineering at the California Institute of Technology, Pasadena, where he is currently an Associate Professor and the Director of the Caltech Micromachining Laboratory. His research interests include MEMS technologies and devices. His current research projects include culture neuron probes, microphones, magnetic actuators, microfluidic systems, and MEMS systems for active fluid control. He is a member of the editorial board of the Journal of Micromechanics and Microengineering.

Dr. Tai has received several honors, including the Presidential Young Investigator Award and the David and Lucile Packard Fellowship. 